\title{
Soluble Tumor Necrosis Factor (TNF)-Like Weak Inducer of Apoptosis (Tweak) Independently Predicts Subclinical Atherosclerosis in Behcet's Disease
}

\author{
Abdullah Icli ${ }^{1}$, Medine Cumhur Cure ${ }^{2}$, Erkan Cure ${ }^{3, *}$, Sevket Arslan ${ }^{4}$, Mehmet Unal ${ }^{5}$, Davut Sakiz \\ Miyase Ozucan, Aysun Toker ${ }^{8}$, Kultigin Turkmen ${ }^{9}$, Adem Kucuk ${ }^{10}$
}

\section{ABSTRACT}

Background: Vasculopathy is a major cause of mortality and morbidity in Behcet's Disease (BD). Subclinical atherosclerosis can even be detected in the early stage of BD. Soluble tumor necrosis factor-like (TNF) weak inducer of apoptosis (TWEAK) is known as a good marker of the inflammation in vascular tree. The aim of this study is to examine the relationship between carotid artery intima-media thickness (cIMT) and serum TWEAK levels in patients with BD.

Materials and Methods: In line with International BD Study Group criteria, 48 BD, and 30 controls were included in our study. Disease activity was evaluated according to BD current activity form (BDCAF). C-reactive protein (CRP), erythrocyte sedimentation rate (ESR), lipid parameters, serum TWEAK levels, and cIMT were measured.

Results: Disease activity score of BD patients was found as 2 (range $0-7)$. cIMT, serum TWEAK, CRP and ESR levels of BD patients were significantly higher comparing to cIMT $(0.62 \pm 0.13 \mathrm{~mm}$ vs. $0.43 \pm 0.09 \mathrm{~mm}, \mathrm{p}<0.001)$, serum TWEAK $(667.5 \pm 130.6 \mathrm{vs} .603 .4 \pm 89.6 \mathrm{pg} / \mathrm{ml}$, $\mathrm{p}=0.015), \operatorname{CRP}(3.9 \pm 4.3 \mathrm{vs} .1 .4 \pm 1.0 \mathrm{mg} / \mathrm{dl}, \mathrm{p}<0.001)$ and $\operatorname{ESR}(10.2 \pm 10.0 \mathrm{vs} .5 .6 \pm 3.7 \mathrm{~mm} / \mathrm{h}, \mathrm{p}=0.005)$ levels of the control group. There was a positive correlation between serum TWEAK level and disease activity $(r=0.251, p=0.030)$ and $c I M T(r=0.463, p<0.001)$. Our study also revealed an independent correlation between cIMT and serum TWEAK levels (beta $=0.354, p<0.001$ ).

Conclusion: Increased serum TWEAK levels can play a part in the development of atherosclerotic heart disease in BD. Due to their liability to atherosclerosis, patients with BD must followed closely.

\section{KEYWORDS}

Behcet's disease; Tumor necrosis factor (TNF)-like weak inducer of apoptosis; TWEAK; carotid artery intima-media thickness; atherosclerosis

\section{AUTHORS AFFILIATIONS}

${ }^{1}$ Department of Cardiology, Necmettin Erbakan University, Konya, Turkey

${ }^{2}$ Department of Biochemistry, Istanbul Laboratory, Istanbul, Turkey

${ }^{3}$ Department of Internal Medicine, Camlica Erdem Hospital, Istanbul, Turkey

${ }^{4}$ Division of Allergy and Clinical Immunology, Department of Internal Medicine, Necmettin Erbakan University, Konya, Turkey

${ }^{5}$ Department of Dermatology, Konya Numune Hospital, Konya, Turkey

${ }^{6}$ Division of Endocrinology, Department of Internal Medicine, Yıldırım Beyazıt Training and Research Hospital, Ankara, Turkey

${ }^{7}$ Department of Internal Medicine, Necmettin Erbakan University, Konya, Turkey

${ }^{8}$ Department of Biochemistry, Necmettin Erbakan University, Konya, Turkey

${ }^{9}$ Division of Nephrology, Department of Internal Medicine, Necmettin Erbakan University, Konya, Turkey

${ }^{10}$ Division of Rheumatology, Department of Internal Medicine, Malatya State Hospital, Malatya, Turkey

* Corresponding Author: Department of Internal Medicine, Camlica Erdem Hastanesi, Uskudar, Istanbul, Turkey; e-mail: erkancure@yahoo.com

Received: 25 May 2018

Accepted: 25 September 2018

Published online: 12 December 2018

Acta Medica (Hradec Králové) 2018; 61(3): 86-92

https://doi.org/10.14712/18059694.2018.123

(c) 2018 The Authors. This is an open-access article distributed under the terms of the Creative Commons Attribution License (http://creativecommons.org/licenses/by/4.0), which permits unrestricted use, distribution, and reproduction in any medium, provided the original author and source are credited. 


\section{INTRODUCTION}

Behcet's disease (BD) is a complex systemic inflammatory disease associated with vascular involvement, thrombogenicity, endothelial cell damage by platelets and leukocytes, and activation of endothelial cells by pro-inflammatory cytokines and chemokines (1). Recent studies still demonstrate the importance of inflammatory cytokines and chemokines because BD affects young adults and it is characterized by remitting-relapsing course (2). Thus, the cardiac involvement is generally associated with morbidity and mortality and it is one of the most severe complications in patients with $\mathrm{BD}(3)$.

Endothelial damage/activation has been described as the initial lesion in the development of atherosclerosis and it has been shown to increase in the active state of disease (4). Furthermore, subclinical atherosclerosis in these patients can be easily and reliably determined by evaluating the carotid artery intima-media thickness (cIMT) (5).

Recent studies emphasize the diagnostic potential of the soluble tumor necrosis factor-like (TNF) weak inducer of apoptosis (TWEAK) as a useful biomarker in various inflammatory and non-inflammatory disorders (6). In recent decades, there has been a significant increase in the literature regarding the use of TNF inhibitors in the treatment of BD. Therefore, TWEAK has been the focus of attention in current studies (7). TWEAK is a cytokine which is mostly derived from leukocytes and belongs to the TNF family. Furthermore, various diseases such as systemic lupus erythematosus (SLE), rheumatoid arthritis (RA), and multiple sclerosis occur through cellular responses which can be associated with inflammatory pathways induced by inflammatory multifunctional cytokines such as TWEAK (8).

Newly mounting evidence has suggested that TWEAK plays a major role in the pathological remodeling underlying other inflammatory diseases, namely cardiovascular diseases and obesity-associated type-2 diabetes mellitus (9-11), particularly in myocardial remodeling leading to heart failure (7). Therefore, soluble TWEAK may be used as a potential biomarker in cardiovascular diseases (12).

In this current study, our aim is to investigate the relationship between serum TWEAK levels and CIMT in patients with BD.

\section{METHODS}

\section{PATIENT POPULATION}

This cross-sectional study was conducted with outpatients who were admitted to the Rheumatology and Cardiology clinics of our University Hospital between May 2015 and October 2015. We included totally 48 male BD patients in this study. Additionally, 30 age- and sex-matched volunteers were selected to be the control group during the same period.

Patients who previously diagnosed with BD according to International BD Study Group criteria were included in this study (International study group for BD) (Evaluation of diagnostic ('classification') criteria in BD disease-towards internationally agreed criteria) (13). Disease severity in BD patients was evaluated by using BD current activ- ity form (BDCAF) (14). Patients with concomitant systemic diseases such as diabetes mellitus, chronic obstructive lung disease, hypertension, coronary artery disease, cancer, thyroid function disorder, hematological disorders, acute or chronic liver and renal diseases, acute or chronic infections, history of smoking and alcohol consumption were excluded from the study. We did not include patients who were actively using drugs such as antihypertensive agents, steroids or statins; which can negatively or positively affect the cIMT values. Subjects with no regular use of medications, smoking history, alcohol consumption and who did not have a known disease were selected as the control group. The study was approved by the Ethics Committee of the Medical Faculty of our university and written and verbal informed consents (consistent with the Helsinki Declaration) were obtained from all participants.

\section{SAMPLE SELECTION (PATIENTS AND CONTROLS)}

Totally 60 outpatients in the Rheumatology clinics were included in this study. However, 12 patients who did not meet the inclusion criteria were excluded from the study. Three out of twelve patients excluded due to hyperlipidemia, 2 of them had a diabetes mellitus, 2 of them had a thyroid disease, 2 of them had a liver dysfunction disease, 1 had a renal disease, 1 had a coronary heart disease, and 1 of them had a hematological disease. Control patients were individuals who were admitted to the cardiology outpatient policlinic in our hospital due to the chest pain and who did not have any cardiac or other diseases as a result of their physical examinations.

\section{CIMT MEASUREMENTS}

In this study, cIMT measurements were performed by one of the authors who were unaware of patients. The Vivid 7 echocardiography device (General Electrics, Horten, Norway) was used to assess the carotid arteries with the help of $10-\mathrm{MHz}$ linear probe. Playback analysis was performed by using the recorded acquired images and they were measured off-line. On both sides of the body, the common carotid artery, the carotid bulb, and internal and external carotid arteries were observed. The intima-media thickness (IMT) was measured in the distal part of the carotid artery which was located in the 15 to $20 \mathrm{~mm}$ proximal to the carotid bulb. Furthermore, two bright echogenic lines, which were located in the arterial wall, were determined as intima and the media. For each side of the body, totally three measurements were performed, mean values of the results were separately calculated for each measurement and findings were recorded for both right and left IMT (15).

\section{SYSTOLIC AND DIASTOLIC BLOOD PRESSURE (BP) MEASUREMENT}

All patients systolic and diastolic BP measurements obtained from their left arm by using sphygmomanometer after 15 minutes relaxing. After two additional recordings which were obtained from the same arm, waiting 5 minutes between two readings, average systolic and diastolic BP were calculated in order to get an accurate measurement. 


\section{BIOCHEMICAL ANALYSIS}

Venous blood samples were collected from all participants after 10-12 h fasting. Fasting serum glucose (FPG), creatinine, alanine aminotransferase (ALT), total cholesterol (TC), triglyceride (TG), high-density lipoprotein cholesterol (HDL-C) and C-reactive protein (CRP) levels were analyzed on Abbot Architect 16000 system with the original reagents. HDL-C levels were detected by using a direct enzymatic method without precipitation. The Friedewald formula was used in order to determine the concentrations of Low-density lipoprotein cholesterol (LDL-C). The method of laser-based flow cytometric impedance was applied to measure the complete blood counts by using an automated blood cell counter (Mindray BC-6800, Shenzhen, PR China). Furthermore, automatic ESR analyzer device was used to measure the erythrocyte sedimentation rate (ESR). On the other hand, commercial kits (Advia Centaur XP System) were used to measure the thyroid stimulating hormone (TSH) levels.

\section{TWEAK MEASUREMENTS}

Enzyme-linked immunosorbent assay kit (eBiosience, Human TWEAK Instant Elisa, Cat no: BMS2006INST) was used in order to measure the serum TWEAK concentrations (expressed as $\mathrm{pg} / \mathrm{mL}$ ) in patients and control individuals. The overall intra-assay coefficient of variation (CV) was calculated as $7.9 \%$.

\section{STATISTICAL ANALYSIS}

Statistical analysis was performed by using the SPSS version 18 (Chicago IL, USA). Results were represented as means \pm standard deviation or median and range depending on data distribution. Normally distributed data were analyzed by using the independent t-test. Pearson correlation test was conducted in order to examine whether or not there is a relationship between cIMT, disease activity, TWEAK levels, and other variables. Stepwise linear regression analysis was conducted in order to detect the variable(s) which mostly affected the dependent variables such as CIMT and disease activity because the numbers of independent variables were quite high. Parameters such as TWEAK levels, systolic and diastolic BP measurements, ESR, CRP, TC, TG, LDL, HDL, age, BMI, the duration of the disease, creatinine, ALT, Hb, WBC, and PLT were independent variables and they were also evaluated. Dependent variables such as cIMT and disease activity were detected by eliminating other step by step with the help of the Stepwise Linear regression analysis. A two-sided $\mathrm{P}<0.05$ was considered significant.

\section{RESULTS}

The mean duration of disease for patients $8.0 \pm 6.3$ years and the disease activity score was 2 (range 0-7). All demographic data of patients with BD were presented in Table 1. cIMT measurements and serum TWEAK levels, as well as CRP and ESR levels of patients with BD, were significant- ly higher comparing to cIMT measurements $(0.62 \pm 0.13$ vs. $0.43 \pm 0.09 \mathrm{~mm}, \mathrm{p}<0.001)$, serum TWEAK $(667.5 \pm 130.6$ vs. $603.4 \pm 89.6 \mathrm{pg} / \mathrm{ml}, \mathrm{p}=0.015)$, CRP (3.9 \pm 4.3 vs. $1.4 \pm 1.0 \mathrm{mg} / \mathrm{dl}, \mathrm{p}<0.001)$, and ESR $(10.2 \pm 10.0$ vs. $5.6 \pm$ $3.7 \mathrm{~mm} / \mathrm{h}, \mathrm{p}=0.005)$ levels of the control group. Also, white blood cell (WBC) count of BD patients $(7.8 \pm 2.8 \times$ $10^{3} / \mathrm{mm}^{3}$ ) was considerably higher than the WBC counts of control individuals $\left(6.6 \pm 1.8 \times 10^{3} / \mathrm{mm}^{3}\right)(\mathrm{p}=0.034)$. Although recordings of systolic and diastolic BP measurements of patients with BD were higher than control group, they were not statistically significant. Table 2 presents ages, BMI values, and biochemical and hematological parameters of patients with $\mathrm{BD}$ and the control group. TWEAK levels were presented in Figure 1.

Tab. 1 Demographics of patients with Behcet's Disease.

\begin{tabular}{|l|l|}
\hline Findings & $\begin{array}{l}\text { Behcet's Disease } \\
(\mathbf{n}=\mathbf{4 8})\end{array}$ \\
\hline Joint involvement $\mathbf{n}(\%)$ & $26(54.1 \%)$ \\
\hline Kidney involvement $\mathbf{n}(\%)$ & $1(2.1 \%)$ \\
\hline Eye involvement $\mathbf{n}(\%)$ & $18(37.5 \%)$ \\
\hline CNS $\mathbf{n}(\%)$ & $2(4.2 \%)$ \\
\hline Pulmonary aneurism $\mathbf{n}(\%)$ & 0 \\
\hline Vascular involvement $\mathbf{n}(\%)$ & $6(12.5 \%)$ \\
\hline Thrombophlebitis $\mathbf{n}(\%)$ & $8(16.7 \%)$ \\
\hline Pathergy $\mathbf{n}(\%)$ & $21(43.8 \%)$ \\
\hline Oral aphthae $\mathbf{n}(\%)$ & $47(97.9 \%)$ \\
\hline Genital ulcer $\mathbf{n}(\%)$ & $19(39.6 \%)$ \\
\hline Folliculitis $\mathbf{n}(\%)$ & $23(47.9 \%)$ \\
\hline Erythema nodosum $\mathbf{n}(\%)$ & $1(2.1 \%)$ \\
\hline GIS involvement (years) $\mathbf{n}(\%)$ & 0 \\
\hline Duration of the disease $\mathbf{n}(\%)$ & $8.0 \pm 6.3$ \\
\hline $\begin{array}{l}\text { Disease activity score median (range) } \\
\text { Colchicine dose (mg) } \\
\text { Azathioprine } \mathbf{n}(\%)\end{array}$ & $2(0-7)$ \\
\hline
\end{tabular}

Abbreviations: CNS - Central Nervous System; GIS - Gastrointestinal System

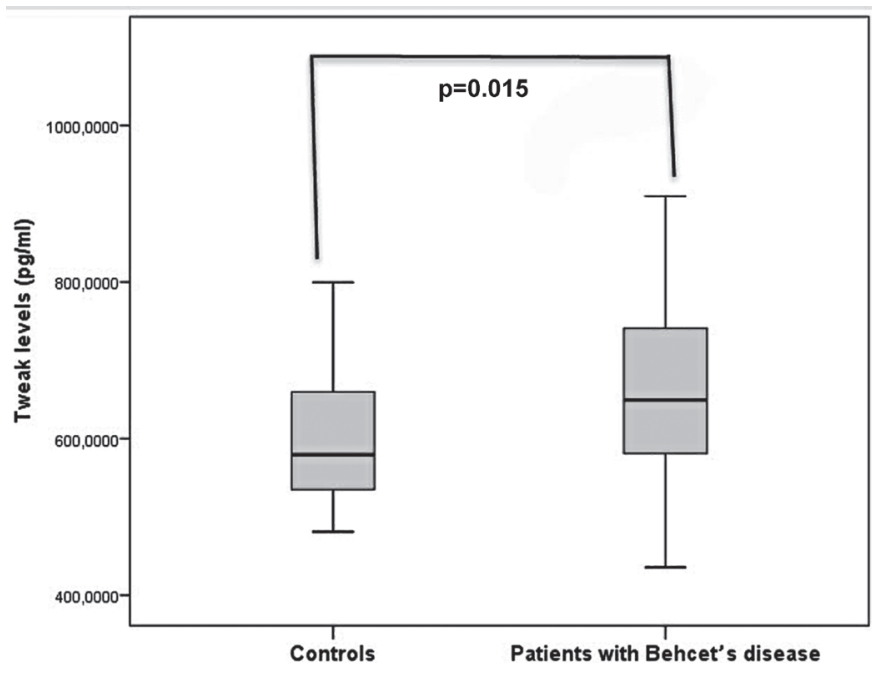

Fig. 1. Serum TWEAK levels in Behcet's disease and control groups. 
Tab. 2 Ages, body mass index, carotid artery intima media thickness, and laboratory parameters of patients with Behcet's disease and the control group.

\begin{tabular}{|c|c|c|c|}
\hline Parameters & $\begin{array}{l}\text { Behcet's } \\
(n=48) \\
(\text { mean } \pm \text { SD) }\end{array}$ & $\begin{array}{l}\text { Control }(n=30) \\
(\text { mean } \pm \text { SD) }\end{array}$ & $P$ value \\
\hline $\begin{array}{l}\text { Age (years) } \\
\text { Gender (M/F) }\end{array}$ & $\begin{array}{l}37.3 \pm 11.2 \\
32 / 16\end{array}$ & $\begin{array}{l}38.2 \pm 12.6 \\
24 / 6\end{array}$ & $\begin{array}{l}0.758 \\
0.155\end{array}$ \\
\hline BMI (kg/m2) & $26.6 \pm 5.0$ & $25.8 \pm 4.5$ & 0.492 \\
\hline cIMT (mm) & $0.62 \pm 0.13$ & $0.43 \pm 0.09$ & 0.001 \\
\hline $\begin{array}{l}\text { Carotid plaque } \\
\text { n (\%) }\end{array}$ & $6(12.5)$ & $0(0)$ & 0.001 \\
\hline SBP (mmHg) & $117.6 \pm 8.6$ & $114.2 \pm 7.5$ & 0.088 \\
\hline $\mathrm{DBP}(\mathrm{mmHg})$ & $72.5 \pm 7.6$ & $69.4 \pm 5.2$ & 0.065 \\
\hline TWEAK (pg/ml) & $667.5 \pm 130.6$ & $603.4 \pm 89.6$ & 0.015 \\
\hline CRP (mg/dl) & $3.9 \pm 4.3$ & $1.4 \pm 1.0$ & 0.001 \\
\hline $\operatorname{ESR}(\mathrm{mm} / \mathrm{h})$ & $10.2 \pm 10.0$ & $5.6 \pm 3.7$ & 0.005 \\
\hline TSH (mU/l) & $1.6 \pm 0.9$ & $1.5 \pm 1.0$ & 0.741 \\
\hline FPG (mg/dl) & $96.1 \pm 15.1$ & $92.9 \pm 5.9$ & 0.198 \\
\hline Creatinine (mg/dl) & $0.79 \pm 0.12$ & $0.85 \pm 0.15$ & 0.099 \\
\hline ALT (IU/L) & $25.2 \pm 16.5$ & $27.1 \pm 17.6$ & 0.663 \\
\hline $\mathrm{TC}(\mathrm{mmol} / \mathrm{l})$ & $4.4 \pm 0.9$ & $4.9 \pm 0.8$ & 0.056 \\
\hline TG (mmol/l) & $1.7 \pm 0.9$ & $1.4 \pm 0.7$ & 0.186 \\
\hline HDL-C (mmol/l) & $1.1 \pm 0.2$ & $1.1 \pm 0.3$ & 0.682 \\
\hline LDL-C (mmol/l) & $2.6 \pm 0.7$ & $2.9 \pm 0.8$ & 0.096 \\
\hline $\mathrm{Wbc}\left(\times 10^{3} / \mathrm{mm}^{3}\right)$ & $7.8 \pm 2.8$ & $6.6 \pm 1.8$ & 0.034 \\
\hline $\mathrm{Hb}(\mathrm{g} / \mathrm{dl})$ & $14.3 \pm 1.5$ & $15.3 \pm 1.8$ & 0.026 \\
\hline $\operatorname{PLT}\left(\times 10^{3} / \mathrm{mm}^{3}\right)$ & $265.7 \pm 57.3$ & $238.8 \pm 63.1$ & 0.073 \\
\hline
\end{tabular}

Abbreviations: BMI - Body mass index; cIMT - Carotid artery intima-media thickness; SBP - systolic blood pressure; DBP - diastolic blood pressure; TWEAK - Tumor necrosis factor (TNF)-like weak inducer of apoptosis; ESR - Erythrocyte sedimentation rate; CRP - C-reactive protein; TSH Thyroid stimulating hormone; FPG - Fasting plasma glucose; ALT - Alanine aminotransferase; TC - Total cholesterol; TG - Triglycerides; HDL-C High-density lipoprotein cholesterol; LDL-C - Low-density lipoprotein cholesterol; Wbc - White blood cells; Hb - Hemoglobin; PLT - Platelets.

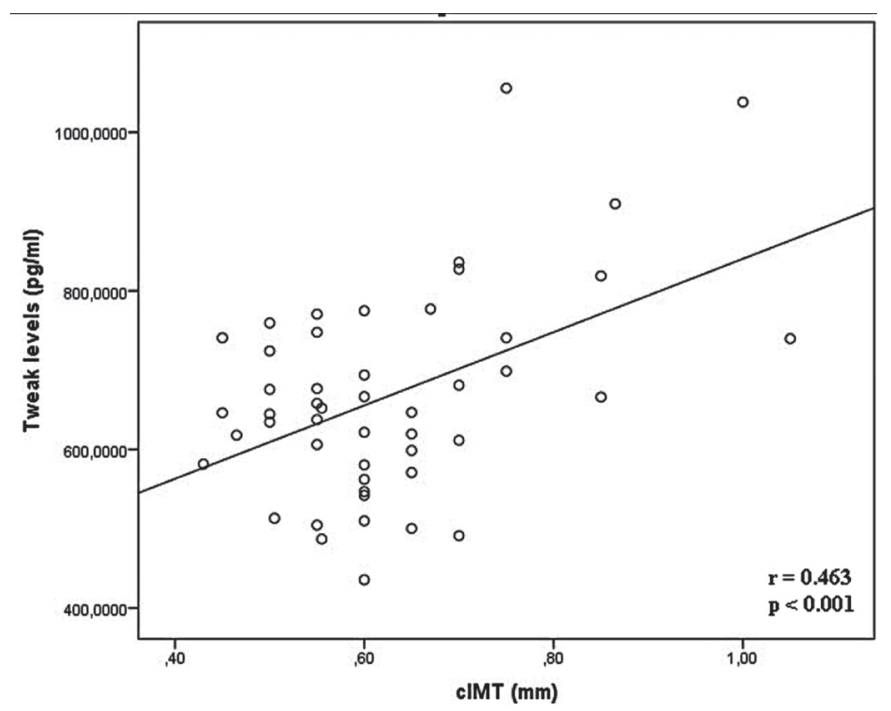

Fig. 2. Correlation between serum TWEAK level and carotid intimamedia thickness.
There was positive correlation between serum TWEAK levels with disease activity $(\mathrm{r}=0.251, \mathrm{p}=0.030)$, cIMT $(\mathrm{r}=0.463, \mathrm{p}<0.001)$, systolic BP $(\mathrm{r}=0.242, \mathrm{p}=0.036)$, diastolic BP recordings $(\mathrm{r}=0.233, \mathrm{p}=0.045)$ and $\mathrm{WBC}$ $(r=0.294, p=0.011)$. There was a positive correlation between cIMT and the disease activity $(r=0.520, p<0.001)$, disease duration $(r=0.455, p<0.001)$, FPG $(r=0.296$, $\mathrm{p}=0.010)$, ESR $(\mathrm{r}=0.259,0.025)$, systolic BP $(\mathrm{r}=0.281$, $\mathrm{p}=0.015)$, and diastolic $\mathrm{BP}(\mathrm{r}=0.249, \mathrm{p}=0.031)$. There was positive correlation between the disease activity and ESR ( $r=0.298, p=0.008), \operatorname{CRP}(r=0.321, p=0.005)$. The cIMT and the disease activity correlation analysis results were presented in Table 3. Correlation relation between cIMT and TWEAK was shown in Figure 2.

Tab. 3 cIMT, disease activity and correlation analysis of other factors (Pearson) in patients with BD.

\begin{tabular}{|l|l|l|l|l|}
\hline \multicolumn{2}{|l|}{ cIMT } & \multicolumn{2}{l|}{ Disease activity } \\
\hline Variable & r value & p value & r value & p value \\
\hline Age & 0.168 & 0.150 & 0.023 & 0.844 \\
\hline BMI & 0.140 & 0.232 & 0.095 & 0.418 \\
\hline Duration of disease & 0.168 & 0.254 & 0.101 & 0.496 \\
\hline Disease activity & $\mathbf{0 . 5 2 0}$ & $\mathbf{0 . 0 0 1}$ & & \\
\hline SBP & $\mathbf{0 . 2 8 1}$ & $\mathbf{0 . 0 1 5}$ & $\mathbf{0 . 1 1 1}$ & $\mathbf{0 . 3 4 4}$ \\
\hline DBP & $\mathbf{0 . 2 4 9}$ & $\mathbf{0 . 0 3 1}$ & $\mathbf{0 . 2 2 3}$ & $\mathbf{0 . 0 5 4}$ \\
\hline TWEAK & $\mathbf{0 . 4 6 3}$ & $\mathbf{0 . 0 0 1}$ & $\mathbf{0 . 2 5 1}$ & $\mathbf{0 . 0 3 0}$ \\
\hline ESR & $\mathbf{0 . 2 5 9}$ & $\mathbf{0 . 0 2 5}$ & $\mathbf{0 . 2 9 8}$ & $\mathbf{0 . 0 0 8}$ \\
\hline CRP & 0.192 & 0.098 & $\mathbf{0 . 3 2 1}$ & $\mathbf{0 . 0 0 5}$ \\
\hline FPG & $\mathbf{0 . 2 9 6}$ & $\mathbf{0 . 0 1 0}$ & 0.171 & 0.142 \\
\hline TSH & 0.075 & 0.523 & 0.112 & 0.317 \\
\hline TC & 0.039 & 0.740 & 0.205 & 0.077 \\
\hline HDL-C & -0.119 & 0.311 & -0.012 & 0.917 \\
\hline TC & 0.118 & 0.314 & 0.048 & 0.682 \\
\hline LDL-C & 0.031 & 0.794 & 0.134 & 0.250 \\
\hline Creatinine & 0.037 & 0.751 & 0.180 & 0.123 \\
\hline ALT & 0.173 & 0.137 & 0.038 & 0.748 \\
\hline WBC & 0.128 & 0.275 & 0.144 & 0.219 \\
\hline Hb & -0.168 & 0.149 & $\mathbf{- 0 . 2 8 1}$ & $\mathbf{0 . 0 1 4}$ \\
\hline Platelets & 0.027 & 0.821 & 0.164 & 0.160 \\
\hline
\end{tabular}

Abbreviations: cIMT - Carotid artery intima-media thickness; BMI - Body mass index; SBP - systolic blood pressure; DBP - diastolic blood pressure; TWEAK - Tumor necrosis factor (TNF)-like weak inducer of apoptosis; ESR Erythrocyte sedimentation rate; CRP - C-reactive protein; FPG - Fasting plasma glucose; TSH - Thyroid stimulating hormone; TC - Total cholesterol; TG - Triglycerides; HDL-C - High-density lipoprotein cholesterol; LDL-C Low-density lipoprotein cholesterol; ALT - Alanine aminotransferase; Wbc - White blood cells; Hb - Hemoglobin.

In stepwise linear regression analysis, we detected an independent relationship between cIMT and the disease activity (beta $[\beta]=0.431, \mathrm{p}<0.001$ ) and TWEAK levels $(\beta=0.354, p<0.001)$ when cIMT was a dependent variable. When disease activity was a dependent variable, there was an independent relationship between disease activity and CRP levels $(\beta=0.297, p=0.008)$ and TWEAK levels $(\beta=$ $0.219, \mathrm{p}=0.040)$. The stepwise regression analysis results were presented in Table 4. 
Tab. 4 Determinants of carotid intima media thickness and disease activity in patients with Behcet's Disease: linear regression analysis.

\begin{tabular}{|l|l|l|l|}
\hline $\begin{array}{l}\text { Dependent } \\
\text { variable }\end{array}$ & $\begin{array}{l}\text { Independent } \\
\text { variables }\end{array}$ & $\begin{array}{l}\text { Beta regression } \\
\text { coefficient }\end{array}$ & P value \\
\hline cIMT & Disease activity & 0.431 & 0.001 \\
\hline & TWEAK levels & 0.354 & 0.001 \\
\hline & & & \\
\hline Disease activity & CRP levels & 0.297 & 0.008 \\
\hline & TWEAK levels & 0.219 & 0.040 \\
\hline
\end{tabular}

Abbreviations: cIMT - carotid intima media thickness; TWEAK -Tumor necrosis factor (TNF)-like weak inducer of apoptosis; CRP - C-reactive protein.

\section{DISCUSSION}

In this present study, we found that patients with $\mathrm{BD}$ have a higher level of cIMT comparing to control group. We also observed carotid plaques in $12.5 \%$ of patients according to their carotid ultrasound imaging. We showed that the serum TWEAK levels in BD patients were significantly higher compared to the TWEAK levels of control group individuals. There was a strong relationship between serum TWEAK levels and both CIMT and the disease activity. In regression analysis, we also detected an independent relationship between cIMT and serum TWEAK levels.

Although the etiology of $\mathrm{BD}$ has not been completely clarified, it is known as an auto-immune disease with chronic inflammation and multi-organ involvement (13). It was reported that auto-immune diseases such as rheumatoid arthritis, SLE, and psoriatic arthritis are characterized with high levels of TWEAK, and disease activity scores of these diseases were also associated with serum TWEAK levels (16-18). TWEAK levels are higher in SLE patients according to the controls and it has been reported that TWEAK levels are highly and positively correlated with the disease activity (19). Furthermore, particularly in SLE patients with vasculitis, serum TWEAK levels were higher compared to the patients without vasculitis (16). TWEAK levels in the synovial fluid of RA patients are also higher compared to the control individuals (20). Besides, serum TWEAK levels were positively correlated with IL-6 and TNF- $\alpha$ levels (17). Also, it has been shown that TWEAK causes the cutaneous vasculitis by leading to the leukocyte migration (21). Low serum TWEAK level was associated with vascular injury in systemic sclerosis which is known as an auto-immune disease (22). During BD, it was detected that pro-inflammatory cytokines such as interleukin (IL) - $1 \beta$, IL-2, IL-8 and TNF-alpha ( $\alpha$ ) increased in all tissues (23). Although some studies claimed that there was a negative relationship between the serum TWEAK levels and IL-6, a number of studies reported that TWEAK simulated the NF- $\mathrm{k} \beta$ nuclear receptor and increased the release of pro-inflammatory cytokines (24-26). In our study, the serum TWEAK levels of BD patients were quite higher compared to control individuals. The increase of the serum TWEAK level can be due to the BD which is a vasculitic disease. Our study is the first study in which the TWEAK levels are examined in $\mathrm{BD}$ patients. Our findings can be beneficial in terms of the role of serum TWEAK levels in $\mathrm{BD}$ patients and the $\mathrm{BD}$ etiology.

TWEAK is a member of the TNF superfamily and has an important role in pro-inflammatory cytokines release, inducing apoptosis, and simulation of cell growth (25-26). In the literature, there are contradictory findings of the relationship between TWEAK levels and atherosclerosis $(27,28)$. Previous studies showed a negative relationship between serum TWEAK levels and coronary artery disease, CIMT, insulin resistance, gestational diabetes and type II diabetes (29-32). However, studies cannot explain the reason why atherosclerotic process accelerated when serum TWEAK levels were low (33). Recently, an increasing number of articles has been reported that increased TWEAK levels are associated with atherosclerosis $(7,8,12)$. It was detected that an increase in serum TWEAK levels resulted in an easy adherence of adhesion molecules to endothelial cells (21). Furthermore, TWEAK levels and increased smooth muscle cell proliferation and an increase in matrix metalloproteinase 3 and 9 activity were reported in experimental studies $(34,35)$. TWEAK was shown to cause prothrombotic events in veins due to the increased release of tissue factor and plasminogen activator in rats, which were injected with the TWEAK (36). Increased release of TWEAK leads to in an increase in pro-inflammatory cytokines and adhesion molecules which results in an increase in the monocyte and macrophage adhesion to arterial walls (37). After the migration of leukocytes, there can be deterioration in the vascular structure and inflammation and vasculitis development can be observed. On the other hand, apoptosis which is increased by TWEAK levels can lead to atherosclerotic heart disease. In other words, TWEAK can lead to vasculitis and atherosclerosis. It has been known that $\mathrm{BD}$ patients have an increased risk of atherosclerotic heart diseases (38). cIMT which is an important marker for subclinical atherosclerosis in BD patients was significantly higher in controls (39). In our study, cIMT and serum TWEAK levels had a strong and independent relationship. TWEAK levels can have an important role in $\mathrm{BD}$ etiopathogenesis and atherosclerotic heart disease development due to $\mathrm{BD}$.

It is well known that hypertension is one of the major factors of atherosclerosis. Ferreira et al. showed that levels of systolic BP were an independent risk factor for cIMT even in normotensive patients (40). In our study patients with history of hypertension were excluded. Although systolic and diastolic BP measurements of patients with BD were within standard normal limits, their recordings were found to be higher compared to control group. As a matter of fact, this difference did not reach statistical significance. There was also a positive correlation between serum TWEAK levels and cIMT. No matter how we observed $\mathrm{BP}$ recordings of patients with $\mathrm{BD}$ were within standard normal limits, there was still a correlation between systolic BP levels and atherosclerosis.

In our study, there was no correlation between the CRP and cIMT whereas there was a weak correlation between ESR and cIMT. However, we found in regression analysis that TWEAK levels mostly affected cIMT. There was a strong relationship between disease activity, CRP, and TWEAK levels. Since high ESR levels in BD patients 
is observed together with high CRP levels in patients with vascular involvement, uveitis, and arthritis. However high ESR levels do not reflect the disease activity in BD patients who do not experience these organ involvements (41). High CRP levels are frequently observed in male BD patients and their high CRP levels reflect the BD disease activity (42). Majority of our patients (2/3) were male and this could be the reason for the significant relationship between CRP levels and the disease activity. Therefore, serum TWEAK levels can be a better marker in BD patients compared to CRP levels in terms of disease activity and atherosclerotic processes. In our study, we detected a strong relationship between cIMT and the disease activity. Therefore, we cannot expect to use TWEAK instead of BDCAF which is a very cheap method in order to mention about the atherosclerotic process in $\mathrm{BD}$ patients. Our study can be a preliminary study which can show the roles of TWEAK levels in BD etiopathogenesis. In this regard, our study can direct the further studies and our findings can be beneficial for the development of new treatment protocols in BD.

\section{LIMITATION OF STUDY}

In our study, the size of our study group was small and we examined only TWEAK levels and cIMT for the detection of atherosclerosis. It could be possible to examine the relationship between increased pro-inflammatory cytokines, TWEAK levels, and other atherosclerosis markers. Furthermore, it could also be possible to have knowledge about the role of TWEAK levels in BD etiopathogenesis by detecting the relationship between $\mathrm{BD}$ patients and other cytokines. Our study is the first one in which TWEAK levels of BD patients were examined and there should be higher numbers of study groups in future studies.

\section{CONCLUSION}

Our study shows that there were higher serum TWEAK levels in patients with $\mathrm{BD}$ compared to control group which may be interpreted as an association between disease activity and atherosclerosis. Even though systolic and diastolic BP recordings of patients with BD were within standard normal limits, there was still a correlation between BP levels and subclinical atherosclerosis. Due to strong relationship between $\mathrm{BD}$ and atherosclerosis and patients with history of BD must follow closely.

\section{FUNDING}

In this study, no funding was received from any of the public or commercial institutions, or for-profit sectors.

\section{REFERENCES}

1. Butta NV, Fernandez-Bello I, Lopez-Longo FJ, Jimenez-Yuste V. Endothelial dysfunction and altered coagulation as mediators of thromboembolism in Behçet Disease. Semin Thromb Hemost 2015; 41: 621-8.

2. Atzeni F, Sarzi-Puttini P, Doria A, Boiardi L, Pipitone N, Salvarani C. Behçet's disease and cardiovascular involvement. Lupus 2005; 14 : 723-6.
3. Ulusan Z, Karadag AS, Tasar M, Kalender M, Darcin OT. Behcet's disease and cardiovascular involvement: our experience of asymptomatic Behcet's patients. Cardiovasc J Afr 2014; 25: 63-6.

4. Merashli M, Ster IC, Ames PR. Subclinical atherosclerosis in Behcet's disease: A systematic review and meta-analysis. Semin Arthritis Rheum 2016;45: 502-10.

5. Yuksel M, Yildiz A, Oylumlu M, et al. Novel markers of endothelial dysfunction and inflammation in Behçet's disease patients with ocular involvement: epicardial fat thickness, carotid intima media thickness, serum ADMA level, and neutrophil-to-lymphocyte ratio. Clin Rheumatol 2016; 35: 701-8.

6. Maecker H, Varfolomeev E, Kischkel F, et al. TWEAK attenuates the transition from innate to adaptive immunity. Cell 2005; 123: 931-44.

7. Novoyatleva T, Sajjad A, Engel FB. TWEAK-Fn14 Cytokine-Receptor Axis: A New Player of Myocardial Remodeling and Cardiac Failure. Front Immunol 2014; 5: 50.

8. Desplat-Jego S, Creidy R, Varriale S, et al. Anti-TWEAK monoclonal antibodies reduce immune cell infiltration in the central nervous system and severity of experimental autoimmune encephalomyelitis. Clin Immunol 2005; 117: 15-23.

9. Burkly LC, Michaelson JS, Hahm K, Jakubowski A, Zheng TS. TWEAKing tissue remodeling by a multifunctional cytokine: role of TWEAK/ Fn14 pathway in health and disease. Cytokine 2007; 40: 1-16.

10. Bertin D, Stephan D, Khrestchatisky M, Desplat-Jego S. Is TWEAK a Biomarker for Autoimmune/Chronic Inflammatory Diseases? Front Immunol 2013; 4: 489.

11. Vendrell J, Chacon MR. TWEAK: A New Player in Obesity and Diabetes. Front Immunol 2013; 4: 488.

12. Blanco-Colio LM, Martín-Ventura JL, Munoz-Garcia B, et al. Identification of soluble tumor necrosis factor-like weak inducer of apoptosis (sTWEAK) as a possible biomarker of subclinical atherosclerosis. Arterioscler Thromb Vasc Biol 2007; 27: 916-22.

13. Criteria for diagnosis of Behcet's disease. International Study Group for Behcet's Disease. Lancet 1990; 335: 1078-80.

14. Bhakta BB, Brennan P, James TE, Chamberlain MA, Noble BA, Silman AJ. Behçet's disease: evaluation of a new instrument to measure clinical activity. Rheumatology (Oxford) 1999; 38: 728-33.

15. Benedetto FA, Mallamaci F, Tripepi G, Zoccali C. Prognostic value of ultrasonographic measurement of carotid intima media thickness in dialysis patients. J Am Soc Nephrol 2001; 12(11): 2458-64.

16. Wang C, Chen LL, Pan HF, Leng RX, Qin WZ, Ye DQ. Expression of human tumor necrosis factor-like weak inducer of apoptosis in patients with systemic lupus erythematosus. Clin Rheumatol 2012; 31(2): 335-9.4

17. Park MC, Chung SJ, Park YB, Lee SK. Relationship of serum TWEAK level to cytokine level, disease activity, and response to anti-TNF treatment in patients with rheumatoid arthritis. Scand J Rheumatol 2008; 37: 173-8.

18. Xia L, Shen H, Xiao W, Lu J. Increased serum TWEAK levels in Psoriatic arthritis: relationship with disease activity and matrix metalloproteinase-3 serum levels. Cytokine 2011; 53: 289-91.

19. Liu ZC, Zhou QL, Li XZ, et al. Elevation of human tumor necrosis factor-like weak inducer of apoptosis in peripheral blood mononuclear cells is correlated with disease activity and lupus nephritis in patients with systemic lupus erythematosus. Cytokine 2011; 53: 295-300.

20. Dharmapatni AA, Smith MD, Crotti TN, et al. TWEAK and Fn14 expression in the pathogenesis of joint inflammation and bone erosion in rheumatoid arthritis. Arthritis Res Ther 2011; 13: R51.

21. Chen T, Guo ZP, Li L, et al. TWEAK enhances E-selectin and ICAM1 expression, and may contribute to the development of cutaneous vasculitis. PLoS One 2013; 8: e56830.

22. Bielecki M, Kowal K, Lapinska A, et al. Diminished production of TWEAK by the peripheral blood mononuclear cells is associated with vascular involvement in patients with systemic sclerosis. Folia Histochem Cytobiol 2009; 47: 465-9.

23. Evereklioglu C, Er H, Türköz Y, Cekmen M. Serum levels of TNF-alpha, sIL-2R, IL-6, and IL-8 are increased and associated with elevated lipid peroxidation in patients with Behçet's disease. Mediators Inflamm 2002; 11: 87-93.

24. Hassan SB, El-demery AB, Ahmed AI, Abukhalil RE. Soluble TWEAK and cardiovascular morbidity and mortality in chronic kidney disease patients. Arab J Nephrol Transplant 2012; 5: 27-32.

25. Campbell S, Michaelson J, Burkly L, Putterman C. The role of TWEAK/ Fn14 in the pathogenesis of inflammation and systemic autoimmunity. Front Biosci 2004; 9: 2273-84.

26. Kim SH, Kang YJ, Kim WJ, et al. TWEAK can induce pro-inflammatory cytokines and matrix metalloproteinase-9 in macrophages. Circ J 2004; 68: 396-9.

27. Gungor O, Kismali E, Sisman AR, et al. The relationships between 
serum sTWEAK, FGF-23 levels, and carotid atherosclerosis in renal transplant patients. Ren Fail 2013; 35: 77-81.

28. Turkmen K, Tonbul HZ, Erdur FM, et al. Soluble TWEAK independently predicts atherosclerosis in renal transplant patients. BMC Nephrol 2013; 14: 144

29. Azak A, Akdoğan MF, Denizli N, et al. Soluble TWEAK levels are independently associated with coronary artery disease severity in patients with stage 2-3 kidney disease. Int Urol Nephrol 2014; 46: 411-5.

30. Vazquez-Carballo A, Ceperuelo-Mallafre V, Chacon MR, et al. TWEAK prevents TNF- $\alpha$-induced insulin resistance through PP2A activation in human adipocytes. Am J Physiol Endocrinol Metab 2013; 305: E101-12.

31. Simon-Muela I, Llaurado G, Chacon MR, et al. Reduced circulating levels of TWEAK are associated with gestational diabetes mellitus. Eur J Clin Invest 2015; 45: 27-35.

32. Kralisch S, Ziegelmeier M, Bachmann A, et al. Serum levels of the atherosclerosis biomarker STWEAK are decreased in type 2 diabetes and end-stage renal disease. Atherosclerosis 2008; 199: 440-4.

33. Blanco-Colio LM, Martín-Ventura JL, Munoz-Garcia B, et al. TWEAK and Fn14. New players in the pathogenesis of atherosclerosis. Front Biosci 2007; 12: 3648-55.

34. Li H, Mittal A, Paul PK, et al. Tumor necrosis factor-related weak inducer of apoptosis augments matrix metalloproteinase 9 (MMP-9) production in skeletal muscle through the activation of nuclear factor-kappaB-inducing kinase and p38 mitogen-activated protein kinase: a potential role of MMP-9 in myopathy. J Biol Chem 2009; 284: 4439-50.
35. Wako M, Ohba T, Ando T, et al. Mechanism of signal transduction in tumor necrosis factor-like weak inducer of apoptosis-induced matrix degradation by MMP-3 upregulation in disc tissues. Spine (Phila Pa 1976)2008; 33: 2489-94.

36. Munoz-Garcia B, Madrigal-Matute J, Moreno JA, et al. TWEAK-Fn14 interaction enhances plasminogen activator inhibitor 1 and tissue factor expression in atherosclerotic plaques and in cultured vascular smooth muscle cells. Cardiovasc Res 2011; 89: 225-33.

37. Blanco-Colio LM. TWEAK/Fn14 Axis: A Promising Target for the Treatment of Cardiovascular Diseases. Front Immunol 2014; 5: 3.

38. Messedi M, Frigui M, Ben Mahfoudh K, et al. Intima-media thickness of carotid artery in patients with Behçet's disease. Arch Med Res 2011; 42: 398-404.

39. Ozturk C, Balta S, Balta I, et al. Neutrophil-lymphocyte ratio and carotid-intima media thickness in patients with Behçet disease without cardiovascular involvement. Angiology 2015; 66: 291-6.

40. Ferreira JP, Girerd N, Bozec E, et al. Intima-Media Thickness Is Linearly and Continuously Associated With Systolic Blood Pressure in a Population-Based Cohort (STANISLAS Cohort Study). J Am Heart Assoc 2016; 5: e003529.

41. Leonardo NM, McNeil J. Behcet's Disease: Is There Geographical Variation? A Review Far from the Silk Road. Int J Rheumatol 2015; 2015: 945262.

42. Müftüoğlu AU, Yazici H, Yurdakul S, et al. Behçet's disease. Relation of serum C-reactive protein and erythrocyte sedimentation rates to disease activity. Int J Dermatol 1986; 25: 235-9. 